\title{
Neural Basis of Acquired Amusia and Its Recovery after Stroke
}

\author{
(iDAleksi J. Sihvonen, ${ }^{1,2}$ (Pablo Ripollés, ${ }^{3,4}$ Vera Leo, ${ }^{2}$ Antoni Rodríguez-Fornells, ${ }^{3,4}$ Seppo Soinila, ${ }^{5}$ \\ and $\odot$ Teppo Särkämö ${ }^{2}$ \\ ${ }^{1}$ Faculty of Medicine, University of Turku, 20520 Turku, Finland, ${ }^{2}$ Cognitive Brain Research Unit, Institute of Behavioural Sciences, University of Helsinki, \\ 00014 Helsinki, Finland, ${ }^{3}$ Cognition and Brain Plasticity Group, Bellvitge Biomedical Research Institute, L’Hospitalet de Llobregat, 08907 Barcelona, Spain, \\ ${ }^{4}$ Department of Basic Psychology, University of Barcelona, 08035 Barcelona, Spain, and ${ }^{5}$ Division of Clinical Neurosciences, Turku University Hospital and \\ Department of Neurology, University of Turku, 20521, Turku, Finland
}

Although acquired amusia is a relatively common disorder after stroke, its precise neuroanatomical basis is still unknown. To evaluate which brain regions form the neural substrate for acquired amusia and its recovery, we performed a voxel-based lesion-symptom mapping (VLSM) and morphometry (VBM) study with 77 human stroke subjects. Structural MRIs were acquired at acute and 6 month poststroke stages. Amusia and aphasia were behaviorally assessed at acute and 3 month poststroke stages using the Scale and Rhythm subtests of the Montreal Battery of Evaluation of Amusia (MBEA) and language tests. VLSM analyses indicated that amusia was associated with a lesion area comprising the superior temporal gyrus, Heschl's gyrus, insula, and striatum in the right hemisphere, clearly different from the lesion pattern associated with aphasia. Parametric analyses of MBEA Pitch and Rhythm scores showed extensive lesion overlap in the right striatum, as well as in the right Heschl's gyrus and superior temporal gyrus. Lesions associated with Rhythm scores extended more superiorly and posterolaterally. VBM analysis of volume changes from the acute to the 6 month stage showed a clear decrease in gray matter volume in the right superior and middle temporal gyri in nonrecovered amusic patients compared with nonamusic patients. This increased atrophy was more evident in anterior temporal areas in rhythm amusia and in posterior temporal and temporoparietal areas in pitch amusia. Overall, the results implicate right temporal and subcortical regions as the crucial neural substrate for acquired amusia and highlight the importance of different temporal lobe regions for the recovery of amusia after stroke.

Key words: amusia; aphasia; music; stroke; voxel-based lesion-symptom mapping; voxel-based morphometry

Significance Statement

Lesion studies are essential in uncovering the brain regions causally linked to a given behavior or skill. For music perception ability, previous lesion studies of amusia have been methodologically limited in both spatial accuracy and time domain as well as by small sample sizes, providing coarse and equivocal information about which brain areas underlie amusia. By using longitudinal MRI and behavioral data from a large sample of stroke patients coupled with modern voxel-based analyses methods, we were able provide the first systematic evidence for the causal role of right temporal and striatal areas in music perception. Clinically, these results have important implications for the diagnosis and prognosis of amusia after stroke and for rehabilitation planning.

\section{Introduction}

Functional and structural neuroimaging studies in healthy subjects have provided evidence for the large-scale involvement of

Received March 3, 2016; revised July 10, 2016; accepted July 12, 2016.

Author contributions: A.R.-F., S.S., and T.S. designed research; A.J.S., V.L., and T.S. performed research; A.J.S., P.R., V.L., and T.S. analyzed data; A.J.S., P.R., V.L., A.R.-F., S.S., and T.S. wrote the paper.

This work was supported by the Academy of Finland program (1257077, 1277693), Turku University Hospital Research Funding, the Finnish Brain Research and Rehabilitation Foundation, the Ella and Georg Ehrnrooth Foundation, the Signe and Ane Gyllenberg Foundation, the Finnish Cultural Foundation, the National Doctoral Programme of Psychology, the Jenny and Antti Wihuri Foundation, the Formación de Profesorado Universitario program (AP2010-4170), and the Generalitat de Catalunya (2014 SGR1413). We thank the staffs of the HUCH Department of Neurology, Turku University Hospital Department of Neurology, and other rehabilitation hospitals in the Hospital District of Southwest Finland and Helsinki metropolitan area for their collaboration. We thank Prof. Mari bilateral temporal, frontal, parietal, and subcortical networks in the neural processing of music (Samson et al., 2010; Zatorre and Salimpoor, 2013; Koelsch, 2014). This evidence is, however,

Tervaniemi, Prof. Riitta Parkkola, Prof. Taina Autti, Dr. Heli Silvennoinen, Jani Saunavaara, PhD, and radiographers Ulla Anttalainen ( $\dagger$ ), Riku Luoto, Pentti Pölönen, and Tuija Vahtera. We also thank the patient subjects and their families for their participation and effort.

The authors declare no competing financial interests.

Correspondence should be addressed to Dr. Aleksi Sihvonen, Cognitive Brain Research Unit, Institute of Behavioural Sciences, Siltavuorenpenger 1 B, Fl-00014 University of Helsinki, Finland. E-mail: ajsihv@utu.fi.

DOI:10.1523/JNEUROSCI.0709-16.2016

Copyright $\odot 2016$ the authors $\quad 0270-6474 / 16 / 368872-10 \$ 15.00 / 0$ 
largely correlational, and lesion-based studies are needed to pinpoint which brain regions are crucial and directly related to music processing. Amusia is a neural disorder characterized by severe impairment of music perception and/or production caused by abnormal brain development (congenital amusia) or brain damage (acquired amusia). Deficit in perceiving fine-grained pitch changes is the hallmark symptom of amusia, but also other domains of music, such as rhythm, can be affected (Stewart et al., 2006).

Although acquired amusia is relatively common after stroke (ranging from 35 to 69\%; Ayotte et al., 2000; Schuppert et al., 2000; Särkämö et al., 2009), the exploration of its neuroanatomical basis has been limited to symptom-led and lesion-led studies of individual cases or small $(N \leq 20)$ patient groups. Acquired amusia has been associated with damage to different temporal, frontal, parietal, and subcortical regions (Stewart et al., 2006), but results regarding lesion lateralization (left/right) and type of musical deficit have been mixed, with some studies reporting spectral (e.g., pitch) or temporal (e.g., rhythm) deficits mainly after right hemisphere damage (Kester et al., 1991; Rosslau et al., 2015) or after both left and right hemisphere damage (Liégeois-Chauvel et al., 1998; Ayotte et al., 2000; Schuppert et al., 2000). Overall, constrained by small sample sizes and low spatial accuracy, these studies provide only coarse information about which specific brain areas are crucial for perceiving the different elements of music.

Voxel-based lesion-symptom mapping (VLSM) is a method for analyzing the relationship between focal brain damage and behavior by using the same voxel-based procedures used in analyzing functional neuroimaging data (Bates et al., 2003). Compared with traditional lesion analysis methods, VLSM is more sophisticated and avoids some of the limitations of classic lesionled or symptom-led approaches (see Materials and Methods). Previously, VLSM has been used with stroke patients to determine lesion-symptom associations related to aphasia (Geva et al., 2012; Henseler et al., 2014; Fridriksson et al., 2015) as well as attention (Grandjean et al., 2008) and motor deficits (Schoch et al., 2006). In the music domain, VLSM has never been used before, whereas voxel-based morphometry (VBM), a method for analyzing gray matter (GM) and white matter (WM) differences between groups or across time (Ashburner and Friston, 2000), has been used in studies of congenital amusia (Hyde et al., 2006, 2007; Mandell et al., 2007; Albouy et al., 2013), musical training (Vaquero et al., 2016), and music-based interventions in stroke patients (Särkämö et al., 2014).

For congenital amusia, VBM and cortical-thickness studies have implicated structural abnormalities in superior temporal and inferior frontal areas as a key mechanism underlying amusia (Hyde et al., 2006, 2007; Mandell et al., 2007; Albouy et al., 2013). However, the results are mixed regarding the lateralization and type (increased/decreased cortical thickness or volume) of these abnormalities, perhaps reflecting the multifaceted nature of congenital amusia (Omigie et al., 2012). Acquired amusia and congenital amusia may stem at least in part from different neural bases, since the latter reflects not just impaired music perception, but a developmental deficit in acquiring musical syntax or a lack of exposure to music (Stewart, 2008). To uncover the brain regions crucial for music perception, systematic and longitudinal research on the neural basis of acquired amusia and its recovery is still needed. Clinically, this information is also important for establishing a more accurate diagnosis and prognosis of amusia and for rehabilitation planning.
Using a large $(N=77)$ sample of stroke patients, the aim of this exploratory combined VLSM-VBM study was to determine the specific lesion patterns associated with acquired amusia and with changes in GM volume (GMV) and WM volume (WMV) related to amusia recovery in a 6 month follow-up.

\section{Materials and Methods}

Subjects and study design. Subjects $(N=77)$ were stroke patients enrolled in two music intervention studies in Helsinki and Turku. Fifty patients were recruited during 2004-2006 from the Department of Neurology of the Helsinki University Central Hospital $(\mathrm{HUCH})$ and 27 patients during 2013-2014 from the Department of Neurology of the Turku University Hospital. All of the patients had an MRI-verified acute ischemic stroke or intracerebral hemorrhage in the left or right hemisphere, primarily in middle cerebral artery (MCA) territory, had cognitive or motor deficits, and were right-handed. All patients had normal hearing. Patients with prior neurological or psychiatric disease or substance abuse were not included. Demographic and clinical characteristics of the patients are presented in Table 1 . The individual studies were approved by the Ethics Committees of the Hospital District of Helsinki and Uusimaa and of the Hospital District of Southwest Finland. Studies were performed in conformance with the Declaration of Helsinki. All patients signed an informed consent and received standard stroke treatment and rehabilitation. All participants underwent a behavioral assessment and an MRI within 3 weeks of the stroke. Behavioral assessment was repeated during the follow-up at 3 months and MRI was repeated at 6 months poststroke.

Behavioral assessment. Music perception was evaluated by using a shortened version (Särkämö et al., 2009) of the Montreal Battery of Evaluation of Amusia (MBEA; Peretz et al., 2003) at the acute stage ( $<3$ weeks after stroke) and at the 3 month poststroke stage as a part of a larger neuropsychological testing battery. We used the MBEA Scale and Rhythm subtests as indices of musical pitch and rhythm perception, respectively, and their average score as an overall index of music perception (referred to hereafter as MBEA total score; Särkämö et al., 2009). Following our previous study (Särkämö et al., 2009) and the established cutoff values of the original MBEA (Peretz et al., 2003), patients with the MBEA total score of $<75 \%$ were classified as amusic [amusic, $N=49$; nonamusic (NA), $N=28$ ]. The amusic and NA groups were relatively well matched demographically and clinically (Table 1), but the amusic group had less education, larger overall lesion volume, and higher incidence of neglect. Importantly, there was no significant difference on the prestroke musical background of the patients. The Rhythm and Scale subtest scores correlated strongly $(r=0.71)$ at the acute stage, and therefore only parametric analyses were carried out.

For the longitudinal VBM analyses (using only the data from the largest cohort of 47 patients from Helsinki), we further subdivided the amusic patients to those who showed and who did not show recovery on the MBEA from the acute to the 3 month stage. Amusic patients with $>20 \%$ increase in MBEA score were classified as recovered amusics (RAs; $N=$ 13; mean, $42 \%$; range, $21-85 \%$ ), amusic patients with a $\leq 20 \%$ increase in MBEA score were classified as nonrecovered amusics (NRAs; $N=16$; mean, $5 \%$; range, -21 to $19 \%$ ), and patients above the acute stage cutoff were classified as NAs $(N=18)$. Overall, the three groups differed with respect to education, lesion volume, and neglect, but there were no differences between the RA and NRA patients. The prestroke musical background of the groups was also comparable. Across patients, changes in the Rhythm and Scale scores from acute to 3 months did not correlate significantly $(r=0.25, p=0.085)$.

To control the specificity of the lesion patterns to amusia, aphasia was also assessed using the Aphasia Severity Rating Scale (ASRS) from the Boston Diagnostic Aphasia Examination (BDAE; Goodglass and Kaplan, 1983). In addition, the performance of the patients in three language tests was used to derive the clinical ASRS estimate: Verbal Fluency Test [listing words from a semantic category (animals); Lezak et al., 2012], shortened Token Test (De Renzi and Faglioni, 1978), and shortened Boston Naming test (Laine et al., 1993).

MRI data acquisition and preprocessing. Patients from Helsinki were scanned with a 1.5T Siemens Vision scanner of the HUCH Department 
Table 1. Demographic and clinical characteristics of the patients

\begin{tabular}{|c|c|c|c|c|c|c|c|}
\hline \multicolumn{4}{|c|}{ VLSM analysis $(n=77)$ of Helsinki and Turku patients } & \multicolumn{4}{|c|}{ VBM analysis ( $n=47)$ of Helsinki patients } \\
\hline & Amusic $(n=49)$ & $\mathrm{NA}(n=28)$ & $p$ value & $\operatorname{NRA}(n=16)$ & $\mathrm{RA}(n=13)$ & NA $(n=18)$ & $p$ value \\
\hline \multicolumn{8}{|l|}{ Demographic } \\
\hline Gender (male/female) & $26 / 23$ & $17 / 11$ & $0.51\left(\chi^{2}\right)$ & $6 / 10$ & $7 / 6$ & $12 / 6$ & $0.24\left(\chi^{2}\right)$ \\
\hline Age (years) & $59.9(10.6)$ & $55.8(10.3)$ & $0.09(t)$ & $62.2(7.7)$ & $58.5(5.4)$ & $56.6(9.9)$ & $0.13(F)$ \\
\hline Education (years) & $10.6(3.7)$ & $13.6(3.1)$ & $<0.001(t)$ & $9.6(2.6)$ & $9.8(4.2)$ & $13.2(3.1)$ & $0.003(F)$ \\
\hline \multicolumn{8}{|l|}{ Music background (prestroke) } \\
\hline Formal music training $^{a}$ & $0.04(0.29)$ & $0.11(0.58)$ & $0.65(U)$ & $0.00(0.00)$ & $0.00(0.00)$ & $0.19(0.68)$ & $0.19(K)$ \\
\hline Instrument playing $^{a}$ & $1.10(1.70)$ & $1.96(2.25)$ & $0.11(U)$ & $1.46(1.86)$ & $1.26(1.94)$ & $1.30(1.81)$ & $0.90(K)$ \\
\hline Music listening prior to stroke ${ }^{b}$ & $4.8(2.0)$ & $3.8(1.8)$ & $0.96(U)$ & $3.3(1.8)$ & $3.4(1.4)$ & $3.9(1.1)$ & $0.61(K)$ \\
\hline \multicolumn{8}{|l|}{ Clinical } \\
\hline Aphasia (no/yes) ${ }^{c}$ & $29 / 20$ & $17 / 11$ & $0.89\left(\chi^{2}\right)$ & $10 / 6$ & $10 / 3$ & $11 / 7$ & $0.61\left(\chi^{2}\right)$ \\
\hline BDAE-ASRS & $4.3(1.1)$ & $4.4(0.9)$ & $0.51(U)$ & $4.3(1.1)$ & $4.5(1.1)$ & $4.3(1.1)$ & $0.89(K)$ \\
\hline MBEA total score percentage & $58.0(8.6)$ & $84.3(6.2)$ & $<0.001(t)$ & $57.8(10.5)$ & $55.2(8.8)$ & $84.9(7.1)$ & $<0.001(F)$ \\
\hline MBEA Rhythm score percentage & $58.1(12.0)$ & $82.0(8.9)$ & $<0.001(t)$ & $60.3(13.8)$ & $56.0(12.3)$ & $84.1(9.7)$ & $<0.001(F)$ \\
\hline MBEA Scale score percentage & $57.8(12.3)$ & $86.6(8.4)$ & $<0.001(t)$ & $55.5(12.6)$ & $54.4(8.5)$ & $85.7(9.2)$ & $<0.001(F)$ \\
\hline$V_{\text {Visual neglect (no/yes) }}^{d}$ & $26 / 18$ & $28 / 0$ & $<0.001\left(\chi^{2}\right)$ & $9 / 7$ & $7 / 6$ & $18 / 0$ & $0.004\left(\chi^{2}\right)$ \\
\hline Other cognitive dysfunction (no/yes) ${ }^{e}$ & $2 / 47$ & $4 / 24$ & $0.11\left(\chi^{2}\right)$ & $0 / 16$ & $1 / 12$ & $2 / 16$ & $0.41\left(\chi^{2}\right)$ \\
\hline Hemiparesis (no/yes) & $11 / 38$ & $8 / 20$ & $0.55\left(\chi^{2}\right)$ & $4 / 12$ & $2 / 11$ & $4 / 14$ & $0.81\left(\chi^{2}\right)$ \\
\hline Lesion laterality (left/right) & $18 / 31$ & $16 / 12$ & $0.08\left(\chi^{2}\right)$ & $8 / 8$ & $3 / 10$ & $10 / 8$ & $0.17\left(\chi^{2}\right)$ \\
\hline Lesion volume in $\mathrm{cm}^{3}$ & $55.8(43.7)$ & $25.5(27.8)$ & $0.001(t)$ & $57.6(46.4)$ & $49.2(35.9)$ & $25.3(31.7)$ & $0.04(F)$ \\
\hline
\end{tabular}

Data are mean (SD) unless otherwise stated. $\chi^{2}, \chi^{2}$ Test; $F$, one-way ANOVA; $K$, Kruskal-Wallis test; $t, t$ test; $U$, Mann-Whitney $U$ test.

${ }^{a}$ Numbers denote values on a Likert scale: $0, n 0 ; 1,<1$ year; $2,1-3$ years; $3,4-6$ years; $4,7-10$ years; $5,>10$ years of training/playing.

${ }^{b}$ Numbers denote values on a Likert scale: range, 0 (never does) to 5 (does daily).

'Classification based on BDAE ASRS: scores $0-4$, aphasia; score 5, no aphasia.

${ }^{d}$ Classification based on the Lateralized Inattention Index of the Balloons Test.

${ }^{e} 0$ ther cognitive dysfunction (attention/executive function or memory deficit).

of Radiology to obtain high-resolution T1 images (flip angle, $15^{\circ}$; TR $=$ $1900 \mathrm{~ms}$; TE $=3.68 \mathrm{~ms}$; voxel size, $1.0 \times 1.0 \times 1.0 \mathrm{~mm}$ ). Patients from Turku were scanned using a 3T Siemens Verio scanner of the Medical Imaging Centre of Southwest Finland. The structural MRI was a T1weighted MPRAGE image (flip angle, $9^{\circ} ; \mathrm{TR}=2300 \mathrm{~ms}$; TE $=2.98 \mathrm{~ms}$; voxel size, $1.0 \times 1.0 \times 1.0 \mathrm{~mm})$.

Normalization of MRI images to a standard template is a necessity for precise comparisons between different subjects or groups, which is especially important when dealing with abnormal brain tissue, such as in stroke patients. To achieve optimal normalization with no postregistration lesion shrinkage or out-of-brain distortion, cost function masking (CFM) was applied (Brett et al., 2001). To define the cost function masks, A.J.S. and T.S. created binary masks of the lesioned areas by manually depicting, on a slice-by-slice basis, the precise boundaries of the lesion directly into the T1 image (Ripollés et al., 2012). An overlap image of all patients' lesions and a statistical power map are shown in Figure 1. The MRIcron software package (http://people.cas.sc.edu/rorden/mricron/ index.html, RRID:SCR_002403) was used to do the aforementioned lesion tracing (Rorden and Brett, 2000).

$\mathrm{T} 1$ images and binary lesion masks were then processed using the Statistical Parametric Mapping software (SPM8, Wellcome Department of Cognitive Neurology, University College London, RRID: SCR_007037) under Matlab 8.0.0 (MathWorks; version R2012b, RRID: SCR_001622). Unified segmentation (Ashburner and Friston, 2005) with medium regularization and CFM was applied to T1 images, segmenting them precisely into GM, WM, and CSF probability maps before normalizing them into the Montreal Neurological Institute (MNI) space. This technique has been widely used with stroke patients (Crinion et al., 2007; Andersen et al., 2010; Ripollés et al., 2012). To preserve the original signal strength during the normalization, GM and WM segmented images were modulated. Smoothing of the tissue probability maps was performed by using an isotropic spatial filter (FWHM, $6 \mathrm{~mm}$ ) to reduce residual interindividual variability. In addition, the lesion masks defined in native space were also registered to MNI space using the normalization parameters obtained during the segmentation process.

Voxel-based lesion symptom mapping. VLSM enables analysis of the relationship between local brain tissue damage and behavioral data in a voxel-by-voxel basis (Bates et al., 2003). By providing a detailed functional map of lesioned brain regions affecting in a given task, VLSM is more sophisticated than traditional lesion analysis, where either a lesionled or a symptom-led approach has been used. In lesion-led studies, patients are grouped on the basis of lesions to a particular brain region and then tested for behavioral differences on a given domain compared with a control group (Chao and Knight, 1998). Although this provides information about the functional roles of the lesioned brain areas, the spatial resolution of this method is coarse and often overlooks lesion areas outside the specific region of interest (areas outside the specific injury site are not considered affecting the behavioral performance and valuable information may be lost). In symptom-led studies, the brain region contributing to the cognitive deficit is being deduced from lesionoverlap images of patients sharing the same behavioral deficit (Dronkers, 1996). To classify patients, this method requires a specific cutoff value on a behavioral task, and parametric analysis is not possible. Thus information of varying performance across a broad spectrum outside cutoff values is lost. As distinct from the previous, VLSM does not require patient grouping by lesion site or behavioral data cut-off, although allows binary variables as well. In three-dimensional lesion maps, formed from MRI images, each voxel is analyzed and the presence, or absence, of a lesion is correlated with the behavioral data. Finally, statistical results and maps of areas associated with the behavioral deficits are shown.

VLSM was performed with nonparametric mapping software (Chris Rorden's NPM, version 6, June 2013) using the normalized acute phase lesion maps for all patients. Parametric analyses were performed using the MBEA total (Scale and Rhythm average) score, Rhythm score, Scale score, and the BDAE-ASRS score. Also, the following binary analyses were performed: amusic versus NA; amusic (no aphasia) versus aphasic (no amusia); amusic versus NA (all aphasics excluded); aphasic versus nonaphasic (all amusics excluded); and amusic versus amusic and aphasic. All voxels damaged $\geq 10 \%$ of the patients were included in the statistical analysis (Dovern et al., 2011; Mirman et al., 2015; Timpert et al., 2015). Correction for multiple comparisons was achieved by using a false discovery rate (FDR)-corrected $p<0.05$ threshold.

Voxel-based morphometry. Morphometric analysis was performed using SPM8. Individual smoothed GM and WM images were entered into a second-level analysis using a Group (RA/NRA/NA) $\times$ Time (Acute/6 months) mixed between-subject and within-subject ANOVA. Six different Group (RA $>$ NRA, RA $>$ NA, NRA $>$ NA) $\times$ Time (Acute $>6$ months, 6 months $>$ Acute) contrasts were calculated. Unless otherwise 


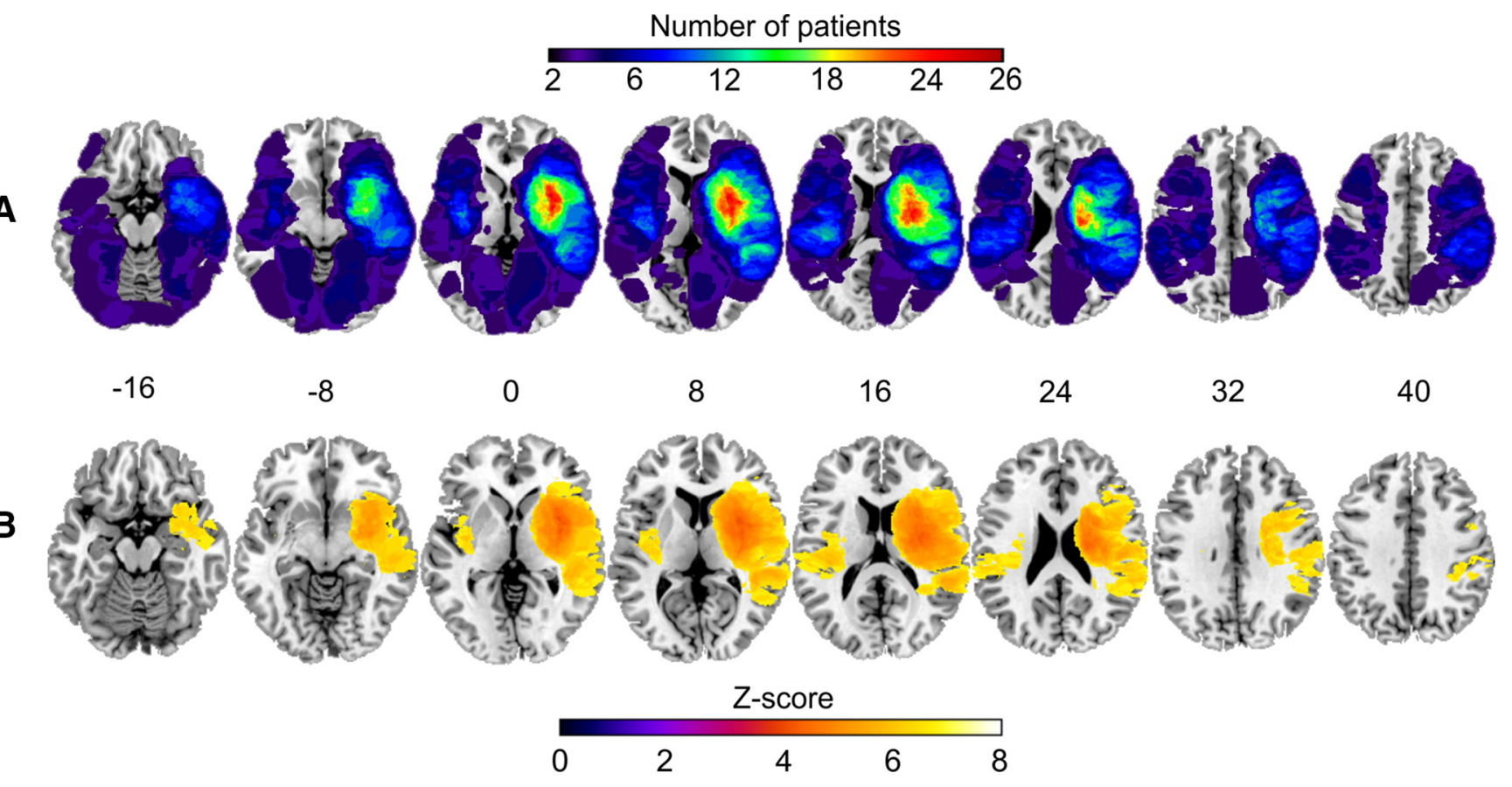

Figure 1. Lesion-overlap maps for all subjects. $A$, Lesion distribution for the whole sample. Warmer areas indicate areas of greater lesion overlap. Accordingly, the color scale ranges from 2 to 26 overlapping subjects. $B$, Statistical power map for VLSM analyses with 77 patients. The map is thresholded at $Z>4$ to view lesion areas with sufficient statistical power to detect an effect of $Z \geq 4$.

noted, results were thresholded at a whole-brain uncorrected $p<0.001$ threshold at the voxel level and a FWE-corrected $p<0.05$ at the cluster level with a cluster extent of $>50$ contiguous voxels. Anatomical and cytoarchitectonical areas were identified using the Automated Anatomical Labeling Atlas (Tzourio-Mazoyer et al., 2002) included in the xjView toolbox (http://www.alivelearn.net/xjview8/, RRID:SCR_008642). As VBM uses voxel intensities and different acquisition settings in different MRI scanners can have a great effect on the results, we used only patients from the Helsinki study with acute and 6 month images $(N=47)$. As stated earlier, patients were from a larger music intervention study (Särkämö et al., 2008), but importantly the intervention and control groups of this previous study did not differ in the recovery from amusia as indexed by change in the MBEA average score (Särkämö et al., 2009). Additionally, the number of NA and amusic patients in the music intervention group was approximately the same (Särkämö et al., 2009). Therefore, the results were not biased in any way by the intervention received.

\section{Results}

Lesion patterns associated with amusia and aphasia

To identify lesion areas associated with amusia and aphasia, we first performed parametric VLSM analyses for the MBEA total scores and BDAE-ASRS scores across all patients. Lower MBEA total scores were associated with lesions in the right superior temporal gyrus (STG), middle temporal gyrus (MTG), Heschl's gyrus (HG), putamen, and insula (Fig. 2A). In contrast, lower BDAE-ASRS scores were associated with lesions in the left STG and insula (Fig. 2B). We then performed binary VLSM analyses directly comparing amusic versus NA patients and aphasic versus nonaphasic patients. As in the parametric analyses, amusia was associated with lesions in the right STG, HG, putamen, and insula (Fig. 2C), and aphasia was associated with lesions in the left STG and insula (Fig. 2D).

To control for the comorbidity of amusia and aphasia ( $41 \%$ of amusics had concurrent aphasia, $65 \%$ of aphasics had concurrent amusia), we performed the same binary analyses first excluding aphasic patients and then comparing pure amusic versus NA patients. Correspondingly, we excluded amusic patients and compared pure aphasic versus nonaphasic patients. This yielded essentially the same results as the first binary analysis, i.e., lesions in the right STG, MTG, HG, putamen, and insula were linked to amusia (Fig. 3A), and lesions in the left $\mathrm{HG}$ and insula were linked to aphasia (Fig. 3B). Similarly, when amusic patients were compared with patients with both amusia and aphasia, lesions in the right STG, MTG, putamen, and insula were again significant (Fig. $3 C$ ). We additionally compared purely amusic (no aphasia) to purely aphasic (no amusia) patients, and lesions associated with amusia comprised yet again the right STG, putamen, and insula, and in aphasia the lesion pattern was localized in the left HG and insula (Fig. 3D).

To determine whether the lesion patterns were similar for pitch and rhythm perception, we performed separate VLSM analyses for the MBEA Scale and Rhythm scores. Parametric analyses (Fig. $4 A, B$ ) indicated a largely overlapping pattern of lesions in the right STG, MTG, HG, insula, and basal ganglia (BG; putamen, caudate, pallidum) for low scores on both Scale and Rhythm subtests (Fig. 4C). Differences in amount of voxels damaged in separate anatomical areas are presented in $\mathrm{Ta}-$ ble 2. The same anatomical areas were seen in both Scale and Rhythm, but the lesions associated with low Rhythm scores covered a larger proportion of the areas. When the lesion pattern associated with poor performance in the Rhythm subtest was further analyzed by limiting the results only to the most significant areas ( $t$ value $>4.5$ ), the right putamen and caudate remained as the most significant components.

\section{GM and WM changes associated with amusia recovery}

The NRAs had greater GMV decrease in the right STG and MTG than the NAs, as shown by a Time (Acute $>6$ months) $\times$ Group (NRA > NA) interaction (Fig. 5A). No significant changes in WM were found in the aforementioned interaction. 
A

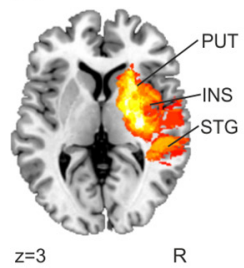

$z=3$

$\mathrm{R}$

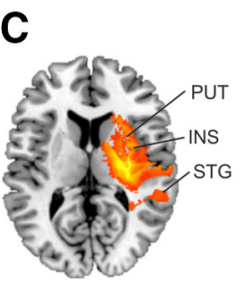

C

$z=11$
MBEA - Parametric

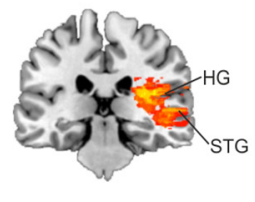

$y=-28 \quad R \quad x=55$

MBEA - Binary

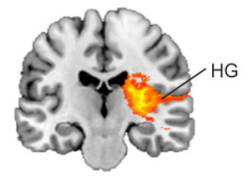

$y=-20$

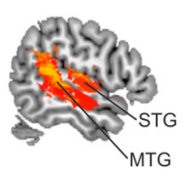

A
B

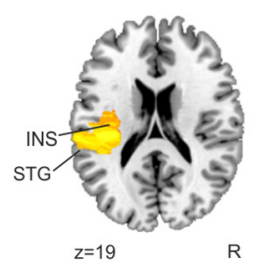

BDAE - Parametric

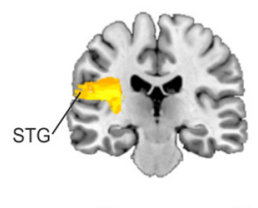

$y=-23$

D

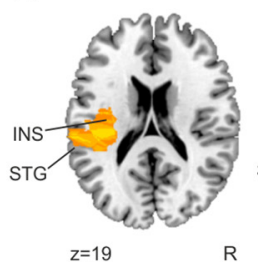

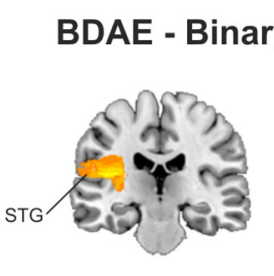

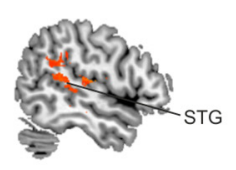

A

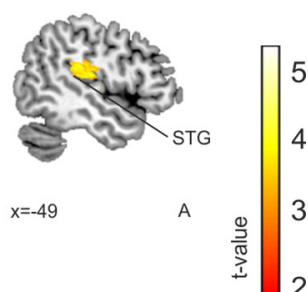

2

Figure 2. VLSM results for amusia and aphasia. $\boldsymbol{A}, \mathrm{MBEA}$ Scale and Rhythm average score (\%). $\boldsymbol{B}, \mathrm{BDAE}-\mathrm{ASRS}$ score. $\boldsymbol{C}$, Amusic versus NA patients. $\boldsymbol{D}$, Aphasic versus nonaphasic patients. Amusia is based on MBEA Scale and Rhythm average score (cutoff, 75\%) and aphasia is based on BDAE-ASRS (cutoff, $<5$ ). $N=77$. Neurological convention is used with MNI coordinates at the bottom left of each slice. All statistical maps are thresholded at a FDR-corrected $p<0.05$ threshold. INS, Insula; PUT, putamen.

A

Amusics vs. Non-amusics (aphasics excluded)

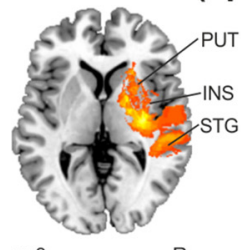

$z=3$

C

$R$
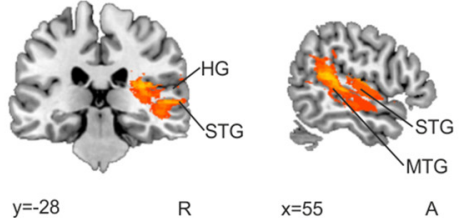

Amusics vs. Aphasics

\& Amusics
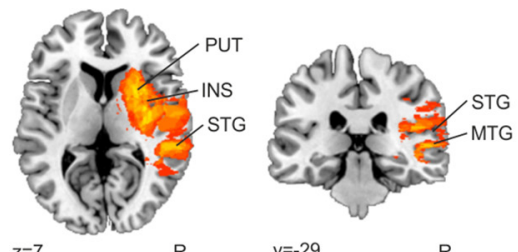

$y=-29$

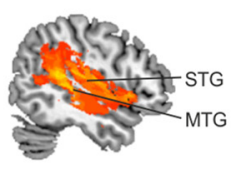

$x=50$
B

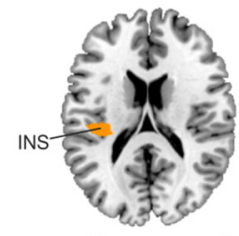

$z=18$
Aphasics vs. Non-aphasics (amusics excluded)

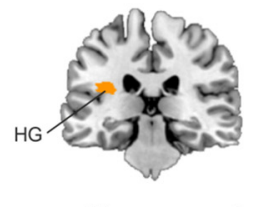

$\mathrm{R}$

$x=-33$

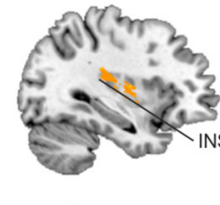

A

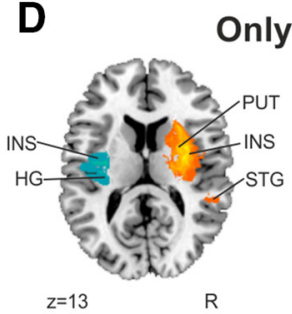

Only Amnsi̊a vs. Only Aphasia

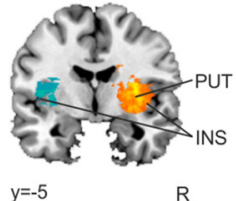

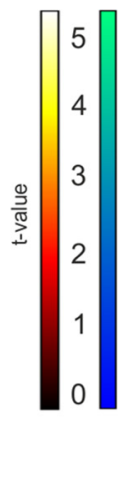

Figure 3. Binary lesion analyses with pure amusics and pure aphasics. $\boldsymbol{A}-\boldsymbol{D}$, Comparisons between $\boldsymbol{A}$, amusic versus NA patients (all aphasic patients excluded; $N=47$ ); $\boldsymbol{B}$, aphasic versus nonaphasic patients (all amusic patients excluded; $N=28$ ); $C$, amusic versus aphasic and amusic patients $(N=49$ ); and $\boldsymbol{D}$, amusic (no aphasia) versus aphasic (no amusia; $N=40$ ). Amusia is based on MBEA Scale and Rhythm average score (cutoff, 75\%) and aphasia is based on BDAE-ASRS (cutoff, $<5$ ). Neurological convention is used with MNI coordinates at the bottom left of each slice. All statistical maps are thresholded at a FDR-corrected $p<0.05$ threshold. INS, Insula; PUT, putamen.

Other contrasts (NRA $>$ RA; RA $>$ NA) did not yield any significant results.

When the two MBEA subtests (Rhythm and Scale) were analyzed separately, we found that in the Rhythm subtest the NRAs showed more GMV decrease in anterior temporal areas than the NAs (Fig. 5B). In this comparison, there was also greater WMV decrease in inferior temporal areas in the NRAs than the NAs (Fig. 5B). In the Scale subtest, the NRAs showed greater GMV decrease in the posterior temporal/temporoparietal area than the NAs [interaction: Time (Acute $>6$ months) $\times$ Group (NRA, Scale $>$ NA, Scale; $p<0.01$ uncorrected; Fig. $5 C$ ]. All significant GMV and WMV decreases are shown in Table 3.

\section{Discussion}

The main finding of the present study was the distinct pattern of lesions in right temporal, insular, and striatal areas underlying acquired amusia after stroke, with additional temporal GMV and WMV decrease observed in patients with persistent amusia. To our best knowledge, this is the first large-scale VLSM-VBM study to systematically explore the neural basis of acquired amusia and its recovery. Importantly, both lesion studies and neuroimaging studies of healthy subjects are needed to better understand the neural systems underlying a given cognitive task (Price and Friston, 2002; Rorden and Karnath, 2004), and our results provide more direct evidence for the role of the aforementioned regions in music perception. Moreover, compared with results from congenital amusics, who are born with a dysfunctional music perception system or are not able to acquire musical syntax during early development, leading to a life-long musical deficit, results from acquired amusia can reveal information about the neuroanatomy of a normally developed and previously functioning music perception system. 


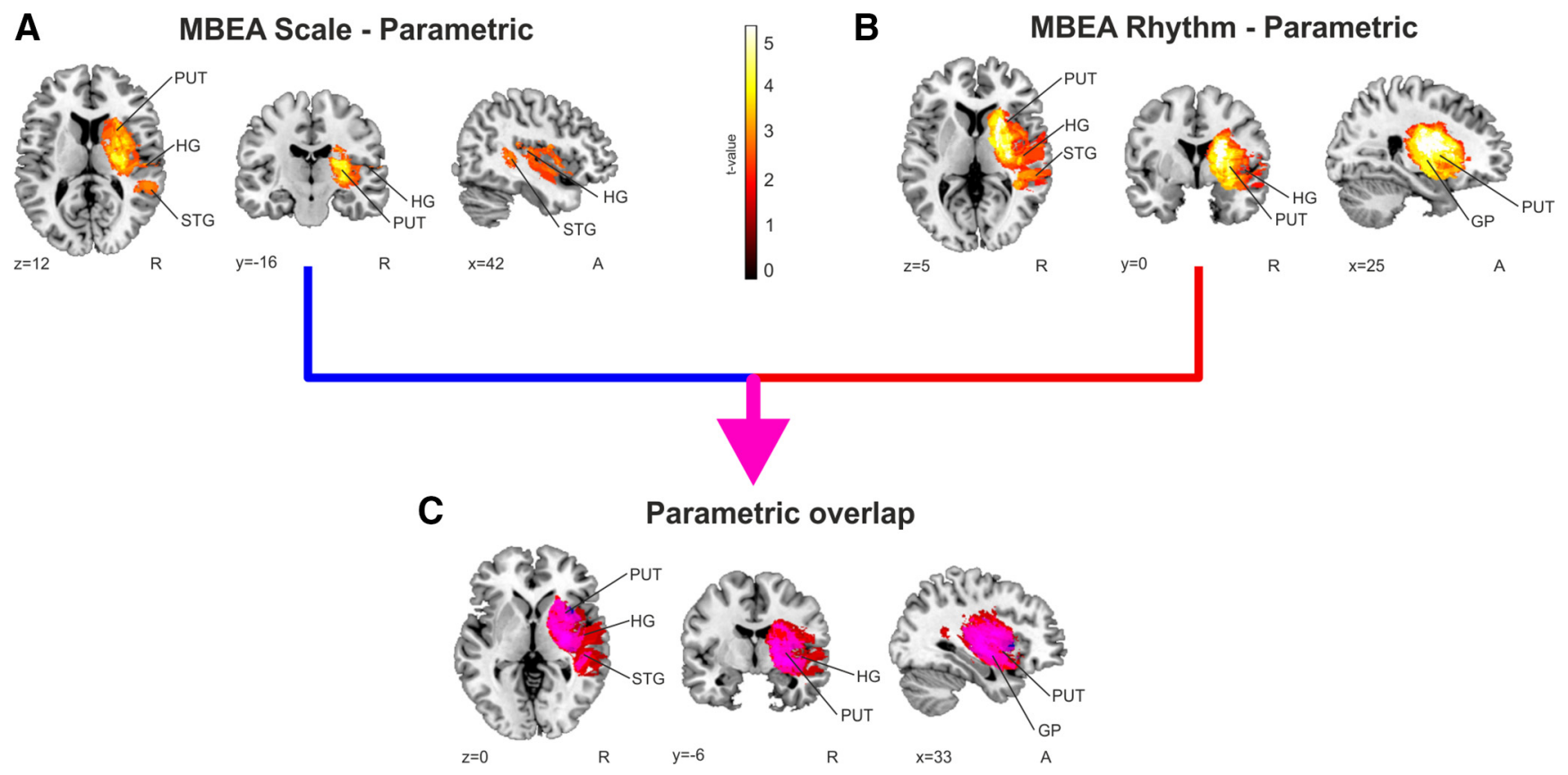

Figure 4. VLSM results for scale and rhythm amusia. $A$, MBEA Scale score. B, MBEA Rhythm score. C, An overlap image comparing Rhythm (red) and Scale (blue) subtests' parametric results $(N=77)$. Neurological convention is used with MNI coordinates at the bottom left of each slice. All statistical maps are thresholded at a FDR-corrected $p<0.05$ threshold. GP, Globus pallidum; PUT, putamen.

Table 2. Anatomical correlates of VLSM results for MBEA Scale and Rhythm scores

\begin{tabular}{lcc}
\hline Anatomical region & Scale & Rhythm \\
\hline Right caudate & $90.02 \%$ & $94.86 \%$ \\
Right HG** & $40.58 \%$ & $90.53 \%$ \\
Right insula* & $38.56 \%$ & $59.23 \%$ \\
Right pallidum & $86.82 \%$ & $89.81 \%$ \\
Right putamen & $90.02 \%$ & $94.86 \%$ \\
Right MTG** & $1.97 \%$ & $18.55 \%$ \\
Right STG** & $11.25 \%$ & $57.04 \%$ \\
Right middle temporal pole & $0.70 \%$ & $1.98 \%$ \\
Right superior temporal pole & $2.70 \%$ & $6.33 \%$ \\
\hline
\end{tabular}

The lesion patterns associated with poor performance in MBEA Scale and Rhythm subtests were overlaid with anatomical masks created with the WFU PickAtlas to obtain the amount of damaged voxels in different anatomical regions. For visual results, see Figure 4.

${ }^{*} p<0.05,{ }^{* *} p<0.005$; comparison $\left(\chi^{2}\right)$ between the percentages of damaged voxels in Rhythm versus Scale (i.e., if the damaged area within the structure is larger in Rhythm than in Scale).

Lesions associated with amusia and lower MBEA total scores were all located in the right MCA territory whereas the lesions associated with aphasia and lower BDAE-ASRS scores were located in the left MCA territory. This is in line with previous group and case studies that have linked the right MCA territory (Ayotte et al., 2000; Rosslau et al., 2015) and especially the right temporal lobe lesions (Mazzoni et al., 1993; Kohlmetz et al., 2003; Terao et al., 2006; Särkämö et al., 2010; Hochman and Abrams, 2014) to acquired amusia. Previous VLSM studies on aphasia have also indicated lesions in the left STG and insula (Geva et al., 2012; Henseler et al., 2014; Fridriksson et al., 2015). In the present sample, $41 \%$ of the amusic patients had at least minor aphasia, which is similar to the percentages (43-55\%) reported in previous amusia case and group studies (Schuppert et al., 2000; Stewart et al., 2006) and suggests that musical and language deficits can often occur together. Although the present sample size prevented us from performing a full $2 \times 2$ analysis [amusia (no/ yes) $\times$ aphasia (no/yes)] or separate analyses within left hemisphere damage (LHD) and right hemisphere damage (RHD) subgroups, we were able to control for the comorbidity of these disorders by performing binary analysis on amusics (no aphasia) versus aphasics (no amusia), amusics versus NAs with all aphasics excluded, aphasics versus nonaphasics with all amusics excluded, and amusics versus amusics and aphasics. Importantly, compared with the primary analyses (Fig. 2), these secondary analyses (Fig. 3) yielded essentially the same key areas, thereby providing further support for the dissociation of the neuroanatomical correlates of amusia and aphasia. This finding is important given the longstanding discrepancy between the results of lesion studies and functional neuroimaging studies of healthy subjects, the former reporting cases of selective impairment of music and language (Sidtis and Volpe, 1988; Piccirilli et al., 2000; Mendez, 2001; for review, see Peretz and Coltheart, 2003) and the latter often reporting significant anatomical overlap in responses to music and speech (Maess et al., 2001; Abrams et al., 2011; Rogalsky et al., 2011; for a recent meta-analysis, see LaCroix et al., 2015). Although our results point to a clear lateralization of amusia and aphasia, music processing is a largely bilateral process, and studies with larger samples of LHD patients are still needed to determine how different left hemisphere regions overlap or separate in amusia and aphasia.

Separate VLSM analyses of the parametric MBEA Scale and Rhythm scores revealed a largely overlapping lesion pattern in the aforementioned right hemisphere network. Naturally, stroke lesions follow the vasculature of the brain and thus are not restricted to a single functional brain area. To pursue the most crucial areas in VLSM results, the $t$-value cutoff can be adjusted to only view the most significant areas in that certain lesion pattern. When this approach was applied to the lesion pattern associated with poor performance in the Rhythm subtest, the right BG remained the most significant element. To our best knowledge, this is the first lesion study to directly implicate the right BG in rhythm perception deficits. In previous studies of healthy subjects, BGs have been linked closely to rhythm analysis (Penhune et al., 1998; Grahn and Brett, 2009; Grahn and Rowe, 2009; Alluri et al., 2012). Recently, professional pianists were also shown to 
GMV differences

in Acute -6 months
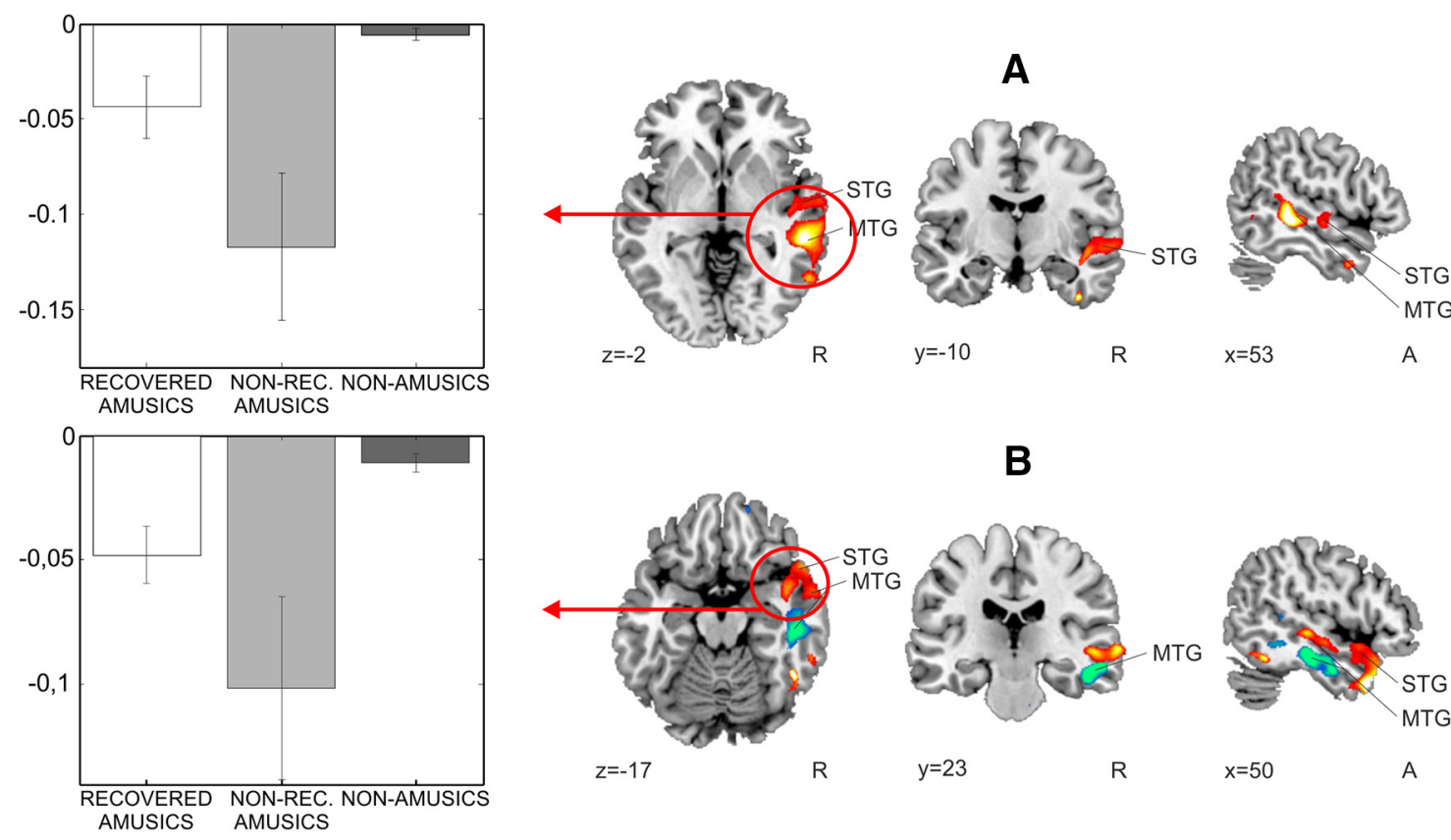

$x=53$

A
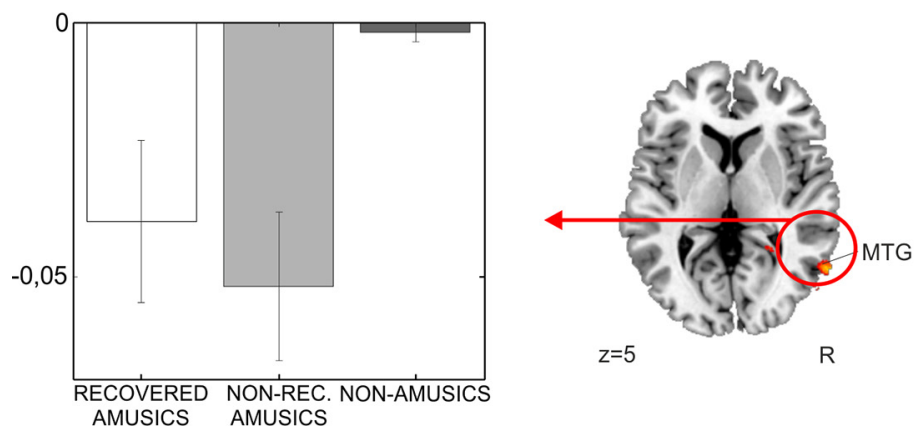

C
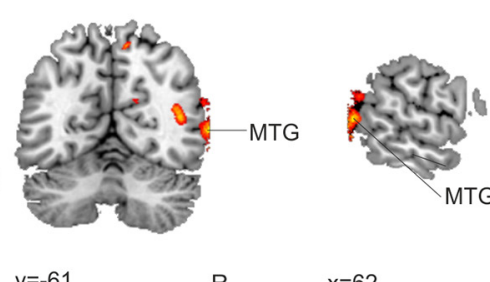

$y=-61$

R

$x=62$

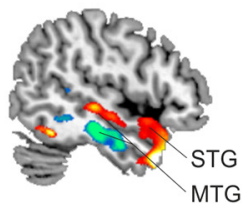

$x=50$

A

Figure 5. VBM results of 47 Helsinki patients. Time (Acute $>6$ months) $\times$ Group (NRAs $>N A s)$ in GMV or WMV interaction. $A, G M N R A s(N=16)$ versus NAs $(N=18) . B, G M($ hot $)$ and WM (cold) nonrecovered rhythm amusics $(N=11)$ versus nonrhythm amusics $(N=18)$. $C$, GM nonrecovered pitch amusics $(N=13)$ versus nonpitch amusics $(N=17)$. Bar plots for $G M V$ differences in Acute $>6$ months. Bar, mean; error bar, SEM. The results are reported at an uncorrected $p<0.001$ threshold at the voxel level (extent threshold: $k>50$ voxels) using MNI coordinates. (lusters for analysis $\boldsymbol{A}$ and $\boldsymbol{B}$ pass a $p<0.05$ FWE-corrected threshold at the cluster level.

Table 3. GMV and WMV decreases (6 month-acute)

\begin{tabular}{|c|c|c|c|}
\hline Anatomical area & MNI coordinates & Cluster size & $t$ value \\
\hline \multicolumn{4}{|l|}{ GM NRAs versus NAs } \\
\hline Right MTG (BA 21) & $54-33-3$ & 8443 & $4.20^{*}$ \\
\hline Right STG (BA 22) & $53-35-4$ & & \\
\hline $\begin{array}{l}\text { Right inferior temporal gyrus } \\
\text { (BA 37) }\end{array}$ & $58-62-6$ & & \\
\hline \multicolumn{4}{|l|}{$\begin{array}{l}\text { GM and WM nonrecovered rhythm } \\
\text { amusics versus nonrhythm } \\
\text { amusics }\end{array}$} \\
\hline Right STG (BA 38) & $47 \quad 17-41$ & 15866 & $4.85^{*}$ \\
\hline Right middle temporal pole & $53 \quad 17-32 ; 650-9$ & & \\
\hline Right MTG WM & $50-24-14$ & 3931 & $4.66^{*}$ \\
\hline Right IFG WM & $49-8 \quad-26 ; 49-17-18$ & & \\
\hline \multicolumn{4}{|l|}{$\begin{array}{c}\text { GM nonrecovered pitch amusics } \\
\text { versus nonpitch amusics }\end{array}$} \\
\hline Right MTG (BA 21) & $63-626$ & 790 & $3.76^{* *}$ \\
\hline Right MTG (BA 19) & $61-6912 ; 63-64-6$ & & \\
\hline
\end{tabular}

For visual VBM results, see Figure 5. IFG, Inferior Temporal Gyrus.

${ }^{*} p<0.05$ FWE-corrected at the cluster level.

${ }^{* *} p<0.01$ uncorrected at the cluster level have skill-related plastic GMV changes in the putamen (Vaquero et al., 2016). Another key area associated with rhythm processing is the cerebellum (Penhune et al., 1998; Sakai et al., 1999); however, due to our inclusion criteria, cerebellar involvement in the rhythm amusia could not be evaluated.

In line with previous studies, we found lesions in the right superior temporal cortex to be associated with pitch amusia (LiégeoisChauvel et al., 1998; Ayotte et al., 2000; Terao et al., 2006; Hochman and Abrams, 2014). Converging evidence from neuroimaging studies of healthy subjects indicates that right superior temporal areas are crucial for pitch and melodic processing (Griffiths et al., 1998; Gutschalk et al., 2002; Patterson et al., 2002; Tramo et al., 2002; Hyde et al., 2008) and that their volume correlates with musical practice (Seither-Preisler et al., 2014) and absolute pitch (Wengenroth et al., 2014). In all of our analyses, right insular lesions were also significantly associated with amusia. Lesions in the insula have previously been linked to pitch amusia after stroke (Terao et al., 2006; Hochman and Abrams, 2014), but may also represent damage to WM fibers in the right frontotemporal pathway, the connectivity of which has been found to be decreased in congenital amusics (Loui et al., 2009; Albouy et al., 2013). 
In the longitudinal VBM analysis, persistent amusia was linked to increasing GMV decline in the right STG, where structural abnormalities have also been observed in congenital amusics (Hyde et al., 2007; Albouy et al., 2013). GM anomalies in congenital amusics are associated with decreased connectivity between the right STG and the right frontotemporal areas (Hyde et al., 2006; Albouy et al., 2013). Correspondingly, we observed a WMV decrease in a region of this same pathway in the NRA patients. Interestingly, further GM atrophy was present in anterior temporal areas in rhythm amusics and posterior temporal areas in pitch amusics. Similar withinhemisphere functional segregation with the anterior STG and superior temporal sulcus showing more sensitivity to changes in the temporal domain and posterior regions showing more sensitivity to changes in the spectral domain has been reported both in animals (Bendor and Wang, 2008) and in humans (Warren et al., 2005; Jamison et al., 2006; for a meta-analysis, see Samson et al., 2010). Also in temporal lobectomy patients, anterior temporal lesions have been linked to deficits in the perception of temporal cues (meter, tempo; Kester et al., 1991; Liégeois-Chauvel et al., 1998).

Another feature of spectral versus temporal processing observed in neuroimaging studies is their lateralization: the right auditory cortex (AC) is more specialized in fine-grained spectral processing and the left $\mathrm{AC}$ in rapid temporal processing of sounds (Zatorre and Belin, 2001; Jamison et al., 2006). However, no clear lateralization effects for musical rhythm processing have been observed in healthy subjects (Samson et al., 2010; Alluri et al., 2012). Meanwhile, lesion studies have provided mixed results, some reporting rhythm or meter discrimination deficits in RHD patients (Kester et al., 1991; Schuppert et al., 2000; Rosslau et al., 2015) or in both LHD and RHD patients (Liégeois-Chauvel et al., 1998; Ayotte et al., 2000). Unfortunately, our sample was not sufficiently powered to detect lesion patterns or GMV/WMV changes for pitch and rhythm amusia separately in LHD and RHD, and larger studies are still needed.

As for the limitations of our study, the groups in the VLSM and VBM studies showed differences on some demographical (education) and clinical (lesion volume, spatial neglect) factors, which may influence the results of the binary analyses. Lower education level (Cox et al., 2006) and larger lesion size (Pan et al., 2006) are associated with higher severity of the stroke, which may lead to a higher likelihood of amusia. The coincidence of neglect and amusia is expected given their similar lesion locations (Chechlacz et al., 2012). Importantly, the RA and NRA patients did not show any significant differences in demographic data. There was only one patient with any formal musical training in our sample. Evaluating the potential facilitating effect of musical training on amusia recovery would call for a larger sample of patients with different levels of musical background. Also, the possible protective effect of higher education in acquired amusia still needs further investigation. Due to the study population (34 LHD vs 43 RHD patients), a mild imbalance was visible in the lesion-overlap image but importantly this difference did not show up as a statistical limitation (Fig. 1): areas in both hemispheres had sufficient statistical power. Because the patients were initially recruited to intervention studies that required some degree of verbal communication and speech comprehension, patients with global or more severe aphasia were in most cases not able to participate, which explains why some of the classical aphasia regions (e.g., Broca's area) do not show up in our VLSM lesion patterns for aphasia.
In conclusion, the results of the present study show that damage to the right temporal areas, insula, and putamen forms the crucial neural substrate for acquired amusia after stroke. Persistent amusia is associated with further atrophy in the right STG and MTG, locating more anteriorly for rhythm amusia and more posteriorly for pitch amusia. It is possible that lesions associated with acquired amusia damage the right frontotemporal pathway, explaining the WMV decrease, possibly leading to neuronal degeneration and consequent GMV decrease. Overall, compared with older lesion studies, these findings provide a more detailed and fine-grained view of the neuroanatomy of amusia and, conversely, highlight which areas are crucial and necessary for normal music perception. In clinical stroke practice, these results have important implications for more accurate identification of amusia, better prediction of the outcome of amusia recovery, and more precise targeting of music-based rehabilitation methods.

\section{References}

Abrams DA, Bhatara A, Ryali S, Balaban E, Levitin DJ, Menon V (2011) Decoding temporal structure in music and speech relies on shared brain resources but elicits different fine-scale spatial patterns. Cereb Cortex 21:1507-1518. CrossRef Medline

Albouy P, Mattout J, Bouet R, Maby E, Sanchez G, Aguera PE, Daligault S, Delpuech C, Bertrand O, Caclin A, Tillmann B (2013) Impaired pitch perception and memory in congenital amusia: the deficit starts in the auditory cortex. Brain 136:1639-1661. CrossRef Medline

Alluri V, Toiviainen P, Jääskeläinen IP, Glerean E, Sams M, Brattico E (2012) Large-scale brain networks emerge from dynamic processing of musical timbre, key and rhythm. Neuroimage 59:3677-3689. CrossRef Medline

Andersen SM, Rapcsak SZ, Beeson PM (2010) Cost function masking during normalization of brains with focal lesions: still a necessity? Neuroimage 53:78-84. CrossRef Medline

Ashburner J, Friston KJ (2000) Voxel-based morphometry-the methods. Neuroimage 11:805-821. CrossRef Medline

Ashburner J, Friston KJ (2005) Unified segmentation. Neuroimage 26: 839-851. CrossRef Medline

Ayotte J, Peretz I, Rousseau I, Bard C, Bojanowski M (2000) Patterns of music agnosia associated with middle cerebral artery infarcts. Brain 123: 1926-1938. CrossRef Medline

Bates E, Wilson SM, Saygin AP, Dick F, Sereno MI, Knight RT, Dronkers NF (2003) Voxel-based lesion-symptom mapping. Nat Neurosci 6:448450. Medline

Bendor D, Wang X (2008) Neural response properties of primary, rostral, and rostrotemporal core fields in the auditory cortex of marmoset monkeys. J Neurophysiol 100:888-906. CrossRef Medline

Brett M, Leff AP, Rorden C, Ashburner J (2001) Spatial normalization of brain images with focal lesions using cost function masking. Neuroimage 14:486-500. CrossRef Medline

Chao LL, Knight RT (1998) Contribution of human prefrontal cortex to delay performance. J Cogn Neurosci 10:167-177. CrossRef Medline

Chechlacz M, Rotshtein P, Humphreys GW (2012) Neuroanatomical dissections of unilateral visual neglect symptoms: ALE meta-analysis of lesion-symptom mapping. Front Hum Neurosci 6:230. CrossRef Medline

Cox AM, McKevitt C, Rudd AG, Wolfe CD (2006) Socioeconomic status and stroke. Lancet Neurol 5:181-188. CrossRef Medline

Crinion J, Ashburner J, Leff A, Brett M, Price C, Friston K (2007) Spatial normalization of lesioned brains: performance evaluation and impact on fMRI analyses. Neuroimage 37:866-875. CrossRef Medline

De Renzi E, Faglioni P (1978) Normative data and screening power of a shortened version of the token test. Cortex 14:41-49. CrossRef Medline

Dovern A, Fink GR, Saliger J, Karbe H, Koch I, Weiss PH (2011) Apraxia impairs intentional retrieval of incidentally acquired motor knowledge. J Neurosci 31:8102-8108. CrossRef Medline

Dronkers NF (1996) A new brain region for coordinating speech articulation. Nature 384:159-161. CrossRef Medline

Fridriksson J, Fillmore P, Guo D, Rorden C (2015) Chronic Broca's aphasia 
is caused by damage to Broca's and Wernicke's areas. Cereb Cortex 25: 4689-4696. CrossRef Medline

Geva S, Baron JC, Jones PS, Price CJ, Warburton EA (2012) A comparison of VLSM and VBM in a cohort of patients with post-stroke aphasia. Neuroimage Clin 1:37-47. CrossRef Medline

Goodglass H, Kaplan E (1983) Boston Diagnostic Aphasia Examination (BDAE). Philadelphia: Lea and Febiger.

Grahn JA, Brett M (2009) Impairment of beat-based rhythm discrimination in Parkinson's disease. Cortex 45:54-61. CrossRef Medline

Grahn JA, Rowe JB (2009) Feeling the beat: premotor and striatal interactions in musicians and nonmusicians during beat perception. J Neurosci 29:7540-7548. CrossRef Medline

Grandjean D, Sander D, Lucas N, Scherer KR, Vuilleumier P (2008) Effects of emotional prosody on auditory extinction for voices in patients with spatial neglect. Neuropsychologia 46:487-496. CrossRef Medline

Griffiths TD, Büchel C, Frackowiak RS, Patterson RD (1998) Analysis of temporal structure in sound by the human brain. Nat Neurosci 1:422-427. CrossRef Medline

Gutschalk A, Patterson RD, Rupp A, Uppenkamp S, Scherg M (2002) Sustained magnetic fields reveal separate sites for sound level and temporal regularity in human auditory cortex. Neuroimage 15:207-216. CrossRef Medline

Henseler I, Regenbrecht F, Obrig H (2014) Lesion correlates of patholinguistic profiles in chronic aphasia: comparisons of syndrome-, modality- and symptomlevel assessment. Brain 137:918-930. CrossRef Medline

Hochman MS, Abrams KJ (2014) Amusia for pitch caused by right middle cerebral artery infarct. J Stroke Cerebrovasc Dis 23:164-165. CrossRef Medline

Hyde KL, Zatorre RJ, Griffiths TD, Lerch JP, Peretz I (2006) Morphometry of the amusic brain: a two-site study. Brain 129:2562-2570. CrossRef Medline

Hyde KL, Lerch JP, Zatorre RJ, Griffiths TD, Evans AC, Peretz I (2007) Cortical thickness in congenital amusia: when less is better than more. J Neurosci 27:13028-13032. CrossRef Medline

Hyde KL, Peretz I, Zatorre RJ (2008) Evidence for the role of the right auditory cortex in fine pitch resolution. Neuropsychologia 46:632-639. CrossRef Medline

Jamison HL, Watkins KE, Bishop DV, Matthews PM (2006) Hemispheric specialization for processing auditory nonspeech stimuli. Cereb Cortex 16:1266-1275. Medline

Kester DB, Saykin AJ, Sperling MR, O’Connor MJ, Robinson LJ, Gur RC (1991) Acute effect of anterior temporal lobectomy on musical processing. Neuropsychologia 29:703-708. CrossRef Medline

Koelsch S (2014) Brain correlates of music-evoked emotions. Nat Rev Neurosci 15:170-180. CrossRef Medline

Kohlmetz C, Müller SV, Nager W, Münte TF, Altenmüller E (2003) Selective loss of timbre perception for keyboard and percussion instruments following a right temporal lesion. Neurocase 9:86-93. CrossRef Medline

LaCroix AN, Diaz AF, Rogalsky C (2015) The relationship between the neural computations for speech and music perception is context-dependent: an activation likelihood estimate study. Front Psychol 6:1138. CrossRef Medline

Laine M, Goodglass H, Niemi J, Koivuselkä-Sallinen P, Tuomainen J, Marttila R (1993) Adaptation of the Boston Diagnostic Aphasia Examination and the Boston Naming Test into Finnish. Scand J Log Phon 18:83-92.

Lezak M, Howieson D, Bigler E, Tranel D (2012) Neuropsychological assessment. New York: Oxford UP.

Liégeois-Chauvel C, Peretz I, Babaï M, Laguitton V, Chauvel P (1998) Contribution of different cortical areas in the temporal lobes to music processing. Brain 121:1853-1867. CrossRef Medline

Loui P, Alsop D, Schlaug G (2009) Tone deafness: a new disconnection syndrome? J Neurosci 29:10215-10220. CrossRef Medline

Maess B, Koelsch S, Gunter TC, Friederici AD (2001) Musical syntax is processed in Broca's area: an MEG study. Nat Neurosci 4:540-545. Medline

Mandell J, Schulze K, Schlaug G (2007) Congenital amusia: an auditorymotor feedback disorder? Restor Neurol Neurosci 25:323-334. Medline

Mazzoni M, Moretti P, Pardossi L, Vista M, Muratorio A, Puglioli M (1993) A case of music imperception. J Neurol Neurosurg Psychiatry 56:322. CrossRef Medline

Mendez MF (2001) Generalized auditory agnosia with spared music recognition in a left-hander. Analysis of a case with a right temporal stroke. Cortex 37:139-150. CrossRef Medline

Mirman D, Chen Q, Zhang Y, Wang Z, Faseyitan OK, Coslett HB, Schwartz
MF (2015) Neural organization of spoken language revealed by lesionsymptom mapping. Nat Commun 6:6762. CrossRef Medline

Omigie D, Müllensiefen D, Stewart L (2012) The experience of music in congenital amusia. Music Percept 30:1-18. CrossRef

Pan SL, Wu SC, Wu TH, Lee TK, Chen TH (2006) Location and size of infarct on functional outcome of noncardioembolic ischemic stroke. Disabil Rehabil 28:977-983. CrossRef Medline

Patterson RD, Uppenkamp S, Johnsrude IS, Griffiths TD (2002) The processing of temporal pitch and melody information in auditory cortex. Neuron 36:767-776. CrossRef Medline

Penhune VB, Zattore RJ, Evans AC (1998) Cerebellar contributions to motor timing: a PET study of auditory and visual rhythm reproduction. J Cogn Neurosci 10:752-765. CrossRef Medline

Peretz I, Coltheart M (2003) Modularity of music processing. Nat Neurosci 6:688-691. CrossRef Medline

Peretz I, Champod AS, Hyde K (2003) Varieties of musical disorders. The Montreal Battery of Evaluation of Amusia. Ann N Y Acad Sci 999:58-75. CrossRef Medline

Piccirilli M, Sciarma T, Luzzi S (2000) Modularity of music: evidence from a case of pure amusia. J Neurol Neurosurg Psychiatry 69:541-545. CrossRef Medline

Price CJ, Friston KJ (2002) Degeneracy and cognitive anatomy. Trends Cogn Sci 6:416-421. CrossRef Medline

Ripollés P, Marco-Pallarés J, de Diego-Balaguer R, Miró J, Falip M, Juncadella M, Rubio F, Rodriguez-Fornells A (2012) Analysis of automated methods for spatial normalization of lesioned brains. Neuroimage 60:12961306. CrossRef Medline

Rogalsky C, Rong F, Saberi K, Hickok G (2011) Functional anatomy of language and music perception: temporal and structural factors investigated using functional magnetic resonance imaging. J Neurosci 31:3843-3852. CrossRef Medline

Rorden C, Brett M (2000) Stereotaxic display of brain lesions. Behav Neurol 12:191-200. CrossRef Medline

Rorden C, Karnath HO (2004) Using human brain lesions to infer function: a relic from a past era in the fMRI age? Nat Rev Neurosci 5:813-819. Medline

Rosslau K, Steinwede D, Schröder C, Herholz SC, Lappe C, Dobel C, Altenmüller E (2015) Clinical investigations of receptive and expressive musical functions after stroke. Front Psychol 6:768. CrossRef Medline

Sakai K, Hikosaka O, Miyauchi S, Takino R, Tamada T, Iwata NK, Nielsen M (1999) Neural representation of a rhythm depends on its interval ratio. J Neurosci 19:10074-10081. Medline

Samson F, Zeffiro TA, Toussaint A, Belin P (2010) Stimulus complexity and categorical effects in human auditory cortex: an activation likelihood estimation meta-analysis. Front Psychol 1:241. CrossRef Medline

Särkämö T, Tervaniemi M, Laitinen S, Forsblom A, Soinila S, Mikkonen M, Autti T, Silvennoinen HM, Erkkilä J, Laine M, Peretz I, Hietanen M (2008) Music listening enhances cognitive recovery and mood after middle cerebral artery stroke. Brain 131:866-876. CrossRef Medline

Särkämö T, Tervaniemi M, Soinila S, Autti T, Silvennoinen HM, Laine M, Hietanen M (2009) Cognitive deficits associated with acquired amusia after stroke: a neuropsychological follow-up study. Neuropsychologia 47: 2642-2651. CrossRef Medline

Särkämö T, Tervaniemi M, Soinila S, Autti T, Silvennoinen HM, Laine M, Hietanen M, Pihko E (2010) Auditory and cognitive deficits associated with acquired amusia after stroke: a magnetoencephalography and neuropsychological follow-up study. PLoS One 5:e15157. CrossRef Medline

Särkämö T, Ripollés P, Vepsäläinen H, Autti T, Silvennoinen HM, Salli E, Laitinen S, Forsblom A, Soinila S, Rodríguez-Fornells A (2014) Structural changes induced by daily music listening in the recovering brain after middle cerebral artery stroke: a voxel-based morphometry study. Front Hum Neurosci 8:245. CrossRef Medline

Schoch B, Dimitrova A, Gizewski ER, Timmann D (2006) Functional localization in the human cerebellum based on voxelwise statistical analysis: a study of 90 patients. Neuroimage 30:36-51. CrossRef Medline

Schuppert M, Münte TF, Wieringa BM, Altenmüller E (2000) Receptive amusia: evidence for cross-hemispheric neural networks underlying music processing strategies. Brain 123:546-559. CrossRef Medline

Seither-Preisler A, Parncutt R, Schneider P (2014) Size and synchronization of auditory cortex promotes musical, literacy, and attentional skills in children. J Neurosci 34:10937-10949. CrossRef Medline 
Sidtis JJ, Volpe BT (1988) Selective loss of complex-pitch or speech discrimination after unilateral lesion. Brain Lang 34:235-245. CrossRef Medline

Stewart L (2008) Fractionating the musical mind: insights from congenital amusia. Curr Opin Neurobiol 18:127-130. CrossRef Medline

Stewart L, von Kriegstein K, Warren JD, Griffiths TD (2006) Music and the brain: disorders of musical listening. Brain 129:2533-2553. CrossRef Medline

Terao Y, Mizuno T, Shindoh M, Sakurai Y, Ugawa Y, Kobayashi S, Nagai C, Furubayashi T, Arai N, Okabe S, Mochizuki H, Hanajima R, Tsuji S (2006) Vocal amusia in a professional tango singer due to a right superior temporal cortex infarction. Neuropsychologia 44:479-488. CrossRef Medline

Timpert DC, Weiss PH, Vossel S, Dovern A, Fink GR (2015) Apraxia and spatial inattention dissociate in left hemisphere stroke. Cortex 71: 349-358. CrossRef Medline

Tramo MJ, Shah GD, Braida LD (2002) Functional role of auditory cortex in frequency processing and pitch perception. J Neurophysiol 87:122-139. Medline

Tzourio-Mazoyer N, Landeau B, Papathanassiou D, Crivello F, Etard O, Delcroix N, Mazoyer B, Joliot M (2002) Automated anatomical la- beling of activations in SPM using a macroscopic anatomical parcellation of the MNI MRI single-subject brain. Neuroimage 15:273-289. CrossRef Medline

Vaquero L, Hartmann K, Ripollés P, Rojo N, Sierpowska J, François C, Càmara E, van Vugt FT, Mohammadi B, Samii A, Münte TF, RodríguezFornells A, Altenmüller E (2016) Structural neuroplasticity in expert pianists depends on the age of musical training onset. Neuroimage 126: 106-119. CrossRef Medline

Warren JD, Jennings AR, Griffiths TD (2005) Analysis of the spectral envelope of sounds by the human brain. Neuroimage 24:1052-1057. CrossRef Medline

Wengenroth M, Blatow M, Heinecke A, Reinhardt J, Stippich C, Hofmann E, Schneider P (2014) Increased volume and function of right auditory cortex as a marker for absolute pitch. Cereb Cortex 24:1127-1137. CrossRef Medline

Zatorre RJ, Belin P (2001) Spectral and temporal processing in human auditory cortex. Cereb Cortex 11:946-953. CrossRef Medline

Zatorre RJ, Salimpoor VN (2013) From perception to pleasure: music and its neural substrates. Proc Natl Acad Sci U S A 110 [Suppl 2]:1043010437. CrossRef Medline 